\title{
A One-Pot Synthesis of $\alpha$-L-Threofuranosyl Nucleoside Triphosphates (tNTPs)
}

\author{
Sujay P. Sau and John C. Chaput \\ Department of Pharmaceutical Sciences, University of California, Irvine, CA 92697-3958, USA.
}

\section{ARTICLE INFO}

Article history:

Received

Revised

Accepted

Available online

Keywords:

TNA triphosphate

phosphoramidite

one-pot synthesis

moisture tolerant

thermal stability

\section{ABSTRACT}

TNA ( $\alpha$-L-threofuranosyl nucleoside) triphosphates of adenosine (tATP), guanosine (tGTP), cytidine (tCTP), and thymidine (tTTP) were synthesized from their corresponding 3'-O phosphoramidite derivatives using a novel one-pot reaction that is less moisture sensitive than traditional methods. The chemically synthesized tNTPs, despite containing an unnatural 3'triphosphate moiety, are similar in thermal stability to natural nucleotide triphosphates.

2009 Elsevier Ltd. All rights reserved.
$\alpha$-L-threofuranosyl-(3' $\rightarrow$ 2') nucleic acid (TNA) is an artificial genetic polymer in which the natural ribose sugar found in RNA has been replaced by an unnatural four-carbon sugar of $\alpha$-L-threofuranose. ${ }^{1}$ This chemical substitution leads to a backbone repeat unit that is one atom shorter than the corresponding backbone repeat unit found in DNA and RNA. ${ }^{2}$ Despite this dramatic structural difference, TNA is capable of forming stable antiparallel Watson-Crick duplexes with itself and with complementary strands of DNA and RNA. ${ }^{3,4}$ The ability to cross-pair with RNA, coupled with the chemical simplicity of threose relative to ribose, has fueled interest in TNA as a progenitor candidate of RNA during a hypothetical period of life's early history known as the RNA world. ${ }^{5,6}$

As part of our effort to explore the chemical etiology of RNA, engineered polymerases have been developed that make it possible to evolve functional TNA molecules in vitro using a selection process that allows genetic information to be copied back and forth between DNA and TNA. ${ }^{7-10}$ Accordingly, TNA molecules isolated from the selection are 'reverse transcribed' into DNA, amplified by PCR, and then 'forward transcribed' back into TNA to generate a new pool of TNA molecules. ${ }^{11}$ Although significant advances have been made toward the engineering of TNA polymerases, ${ }^{12,13}$ the process of in vitro selection (SELEX) remains limited by the low availability of TNA triphosphates. ${ }^{14}$ To overcome this bottleneck, we developed an optimized synthesis protocol for generating TNA nucleosides, which are the chemical precursors of TNA phosphoramidites and triphosphates used to produce TNA polymers by solid-phase and enzymatic synthesis, respectively. ${ }^{15}$ The new synthetic protocol involves ten chemical transformations with three crystallization steps and a single silica gel chromatography step to afford the desired molecules in an overall yield of $\sim 16-23 \%$ depending on the identity of the nucleobase.

TNA triphosphates have been synthesized previously using the Ludwig-Eckstein method from their corresponding 2'-ODMT protected nucleosides. ${ }^{16,17}$ This method was necessary as the traditional Yoshikawa method with $\mathrm{POCl}_{3}$ proved difficult due to steric hindrance of the 3'-hydoxyl group and potential loss of the acid-labile DMT group. ${ }^{18}$ Unlike most other nucleoside triphosphates, which derive from ribose or deoxyribose sugars and therefore require phosphorylation at the 5'-hydroxyl position, TNA triphosphate synthesis requires phosphorylating a secondary hydroxyl group at the 3' position of the sugar ring. The alcohol moiety at this position is less reactive than a typical secondary hydroxyl group due its close proximity to the protected nucleobase.
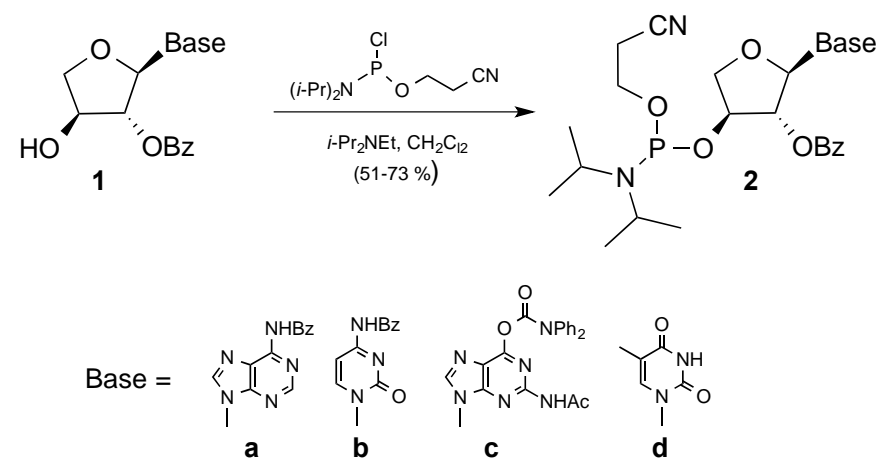

Scheme 1. Synthesis of 3'- $O$-phosphoramidite derivatives of $\alpha$-Lthreofuranosyl nucleosides. 
Although the Ludwig-Eckstein method represents a valuable strategy for generating TNA triphosphates, ${ }^{17}$ we wished to develop new methodology that was less moisture sensitive and more amenable to preparative HPLC purification. In our experience, traditional methods produce complex HPLC chromatograms that make it difficult to isolate the desired product from the reaction mixture. While this problem is less severe when the reaction is performed under anhydrous conditions, we felt that such constraints placed undue limitations on the level of expertise required to generate tNTP substrates.

Here, we describe a novel one-pot synthesis of TNA nucleoside triphosphates bearing all four genetic bases $(A, C, T, G)$ from their corresponding 3'- $O$-phosphoramidite precursors. The reaction is water tolerant and leads to the formation of two major peaks (nucleotide monophosphate and triphosphate) that are easily separated by HPLC chromatography. Using this approach, milligram quantities of highly pure TNA triphosphate can be generated in just a few days.

Starting from previously described 2'-O-benzoyl protected TNA nucleosides 1a-d, ${ }^{15}$ the corresponding 3'- $O$-phosphoramidite derivatives 2a-d were synthesized upon treatment with 2cyanoethyl- $N, N$-diisopropylchlorophosphine (Scheme 1). Under standard reaction conditions, phosphitylation proceeded very slowly and was incomplete after overnight incubation. However, the inclusion of 4-dimethylaminopyridine (DMAP) as nucleophilic catalyst allowed the reaction to go to completion in 2.5 hours. Following purification by silica gel chromatography, nucleosides 2a-d are obtained in $51-73 \%$ yield.

Synthesis of TNA triphosphates from 2a-d follows a path in which each phosphoramidite is hydrolyzed to H-phosphonate, in situ oxidized to the chlorophosphate, and then treated with inorganic pyrophosphate to obtain the protected triphosphate 5ad (Scheme 2). In a single one-pot reaction, hydrolysis of $\mathbf{2 a - d}$ in presence of ethylthiotetrazole as an activating agent provided the corresponding H-phosphonate derivatives 3a-d. Subsequent oxidation and pyrophosphate coupling was achieved by the stepwise addition of two acetonitrile solutions containing $\mathrm{N}$ chlorosuccinimide (NCS) and bis(tri- $n$-butyl-amine) dihydrogen pyrophosphate. Analysis of the crude reaction mixture by MALDI-TOF mass spectrometry indicated that the TNA triphosphate species 5a-d formed in each case.

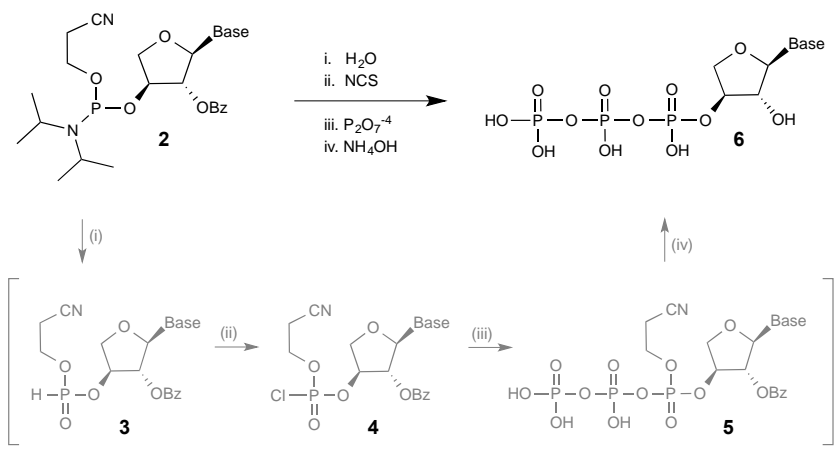

Scheme 2. Synthesis of $\alpha$-L-threofuranosyl nucleoside triphosphates (tNTPs) from 3'-O-phosphoramidites.

TNA triphosphates 6a-d were obtained by treatment with conc. ammonia in water for $16 \mathrm{~h}$ at $37{ }^{\circ} \mathrm{C}$. After deprotection, ion-pair reverse-phase HPLC analysis of the crude reaction mixture revealed two major peaks in the chromatogram that corresponded to the nucleoside monophosphate (major peak) and desired TNA triphosphate (minor peak) (Figure S1). The identity of the desired TNA triphosphates 6a-d was confirmed by coinjection with authentic samples and mass spectrometry analysis. Each TNA triphosphate was purified by single injection on a preparative reverse-phase HPLC column. The generality of the reaction was demonstrated by synthesizing all four tNTPs with isolated yields of $8-13 \%$ with $>90 \%$ purity (Figure 1 ).

To investigate the reaction chemistry, we monitored product formation during the course of the reaction by ${ }^{31} \mathrm{P}$ NMR. In the absence of pyrophosphate, oxidation of the H-phosphonate intermediate 3a produced a dinucleotide pyrophosphate intermediate ( $-14.5 \mathrm{ppm}$, multiplet). In presence of added excess water, the dinucleotide intermediate decomposed to the nucleoside monophosphate byproduct $(-2.5 \mathrm{ppm}$, singlet) (Figure $\mathrm{S} 2$ ), which explains the origin of the nucleotide monophosphate in the crude reaction mixture.

Similar results were also observed by HPLC analysis. In this case, deprotection of the tTTP monomer following oxidation and phosphorylation with $\left(\mathrm{Bu}_{3} \mathrm{NH}\right)_{2} \mathrm{H}_{2} \mathrm{P}_{2} \mathrm{O}_{7}$ yielded a third peak in the HPLC trace (Figure S3) that was attributed to the formation of a dinucleoside pyrophosphate intermediate. HPLC analysis of the deprotection reaction after 2 and 18 hours of treatment with $33 \%$ aqueous $\mathrm{NH}_{4} \mathrm{OH}$ reveals a marked decrease in the intermediate peak with a concomitant increase in the peak corresponding to the thymidine monophosphate byproduct. This result is consistent with the unwanted formation of a dinucleotide by-product that degrades to form the TNA nucleoside monophosphate.

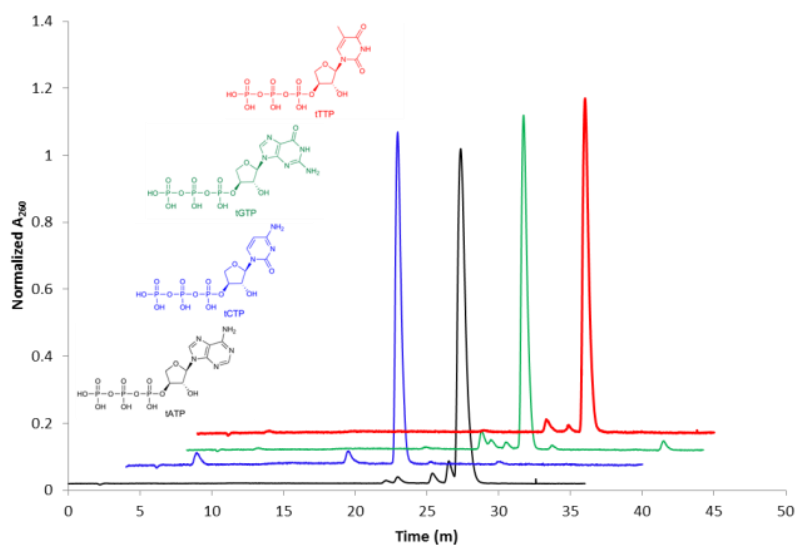

Figure 1. Analytical HPLC chromatogram of purified tNTPs.

In an attempt to improve the yield of triphosphate synthesis, we explored several additives that could be used to trap the chlorophosphate intermediates $\mathbf{4 a - d}$ as a less reactive species. Unfortunately, none of the additives tested (which included imidazole, 1,2,3-triazole, 1,2,4-triazole, benzotriazole, and $\mathrm{N}$ hydroxy benzotriazole) reduced the yield of monophosphate formation. In each case, HPLC analysis showed a distribution of nucleoside monophosphate to triphosphate that was similar to what was observed in original reaction (Figure S1), which is consistent with our observation that dinucleotide formation outcompetes triphosphate formation. Nevertheless, the ability to make TNA triphosphates under non-anhydrous conditions is valuable for routine chemical synthesis.

Since the main benefit of our strategy is the ability to synthesize TNA triphosphates under conditions that are tolerant to moisture, we examined the reaction in the presence of increasing amounts of water. To our surprise, the addition of 75 molar equivalents of water $(6 \% \mathrm{v} / \mathrm{v}$ water to $\mathrm{MeCN})$ did not significantly change the product distribution. This result 
demonstrates that although the overall reaction yields are modest (8-13\% purified yield), the potential to perform phosphorylation chemistry under non-anhydrous conditions is considerable.

To our knowledge, TNA triphosphates are the only known substrates for a polymerase reaction where the triphosphate moiety is located on a secondary hydroxyl group on the sugar ring. Despite numerous reports of polymerase-mediated TNA synthesis, ${ }^{7-12}$ at present, no information is available on the thermal stability of TNA triphosphates. To emulate standard laboratory usage, we analyzed the stability of TNA triphosphates over a range of temperatures and freeze-thaw cycles. This included polymerase reaction conditions for extended periods of time at $55^{\circ} \mathrm{C}$ and at elevated temperatures of 75 and $95^{\circ} \mathrm{C}$ as well as iterative rounds of heating and cooling in a simulated polymerase chain reaction (PCR). As shown in Table 1, >95\% of the tNTPs remain intact after 5 hours of simulated polymerase extension at $55^{\circ} \mathrm{C}$ and after 10 iterative cycles of freezing and thawing. Similarly, $>75 \%$ of the tNTPs are present after 30 cycles of PCR. In fact, marked degradation only occurs when the samples are heated for $5 \mathrm{~h}$ at elevated temperatures. Similar results were also obtained for dATP and ATP, indicating that tNTPs are similar in thermal stability to natural nucleoside triphosphates.

Table 1. Thermal stability of tNTPs under various conditions.

\begin{tabular}{lllllll}
\hline \multirow{2}{*}{ Conditions } & \multicolumn{5}{c}{ Triphosphate Remaining (\%) } \\
\cline { 2 - 7 } & tTTP & tGTP & tCTP & tATP & ATP & dATP \\
\hline 10 cycles of freeze-thaw* & 98.7 & 99.8 & 98.1 & 96.7 & nd & Nd \\
10 cycles of PCR & 91.5 & 87.8 & 90.7 & 86.5 & 93.7 & 94 \\
20 cycles of PCR & 88.7 & 82.9 & 86.2 & 81.8 & 90.5 & 89.9 \\
30 cycles of PCR & 85.2 & 77.5 & 82 & 77.2 & 86.6 & 86.4 \\
$55^{\circ} \mathrm{C}$ 1h & 99.4 & 98.5 & 99.6 & 96.4 & 99.3 & 99.9 \\
$55^{\circ} \mathrm{C}$ 5h & 97.3 & 96.1 & 97.4 & 93.4 & 93.5 & 93.6 \\
$55^{\circ} \mathrm{C}$ 25h & 84.2 & 83.5 & 89.6 & 76.2 & nd & nd \\
$75^{\circ} \mathrm{C}$ 5h & 71.8 & 65.9 & 68.5 & 65.4 & 71.2 & 70.4 \\
\hline
\end{tabular}

Chemical stability was analyzed by ion-pair reverse-phase HPLC on C18 column $(150 \times 4.6 \mathrm{~mm})$ with a gradient $(0-10 \%$ over $40 \mathrm{~m})$ of acetonitrile in $0.1 \mathrm{M}$ triethylammonium acetate buffer. All samples contained at $0.1 \mathrm{mM}$ tNTP in $1 \mathrm{x}$ thermopol buffer supplemented with $1 \mathrm{mM} \mathrm{MnCl}_{2}$. *Freeze thaw cycles were performed with $2 \mathrm{mM}$ tNTP in water. n.d.= not determined.

In summary, we describe a new method for synthesizing TNA nucleoside triphosphates that does not require anhydrous conditions and enables rapid purification by reverse-phase HPLC chromatography. Given the recent interested in developing TNA aptamers for synthetic biology, ${ }^{19}$ we suggest that this new procedure will be useful in releasing the current bottleneck in TNA triphosphate synthesis.

\section{Acknowledgments}

We wish to thank members of the Chaput laboratory for their helpful comments and suggestions. This work was supported by the DARPA Folded Non-Natural Polymers with Biological Function Fold F(x) Program under award number N66001-14-24054 and by grants from the National Science Foundation (1615804 and 1607111).

\section{References and notes}

1. Schoning, K. U.; Scholz, P.; Guntha, S.; Wu, X.; Krishnamurthy, R.; Eschenmoser, A. Science 2000, 290, 1347.

2. Ebert, M.-O.; Mang, C.; Krishnamurthy, R.; Eschenmoser, A.; Jaun, B. J. Am. Chem. Soc. 2008, 130, 15105.

3. Schoning, K.-U.; Scholz, P.; X., W.; Guntha, S.; Delgado, G.; Krishnamurthy, R.; Eschenmoser, A. Helv. Chim. Acta 2002, 85, 4111.

4. Yang, Y.-W.; Zhang, S.; McCullum, E. O.; Chaput, J. C. J. Mol. Evol. 2007, 65, 289 .

5. Orgel, L. E. Science 2000, 290, 1306

6. Joyce, G. F. Nature 2002, 418, 214.

7. Chaput, J. C.; Szostak, J. W. J. Am. Chem. Soc. 2003, 125, 9274.

8. Chaput, J. C.; Ichida, J. K.; Szostak, J. W. J. Am. Chem. Soc. $\mathbf{2 0 0 3}, 125,856$.

9. Kempeneers, V.; Vastmans, K.; Rozenski, J.; Herdewijn, P. Nucleic Acids Res. 2003, 31, 6221.

10. Pinheiro, V. B.; Taylor, A. I.; Cozens, C.; Abramov, M.; Renders, M.; Zhang, S.; Chaput, J. C.; Wengel, J.; Peak-Chew, S. Y.; McLaughlin, S. H.; Herdewijn, P.; Holliger, P. Science 2012, 336, 341.

11. Yu, H.; Zhang, S.; Dunn, M.; Chaput, J. C. J. Am. Chem. Soc. 2013, 135, 3583 .

12. Larsen, A. C.; Dunn, M. R.; Hatch, A.; Sau, S. P.; Youngbull, C.; Chaput, J. C. Nature Commun. 2016, In press.

13. Dunn, M. R.; Otto, C.; Fenton, K. E.; Chaput, J. C. ACS Chem. Biol. 2016, 10.1021/acschembio.5b00949.

14. Yu, H.; Zhang, S.; Chaput, J. C. Nat. Chem. 2012, 4, 183

15. Sau, S. P.; Fahmi, N. E.; Liao, J.-Y.; Bala, S.; Chaput, J. C. J. Org. Chem. 2016, 81, 2302.

16. Zou, K.; Horhota, A.; Yu, B.; Szostak, J. W.; McLaughlin, L. W. Org. Lett. 2005, 7, 1485.

17. Ludwig, J.; Eckstein, F. J. Org. Chem. 1989, 54, 631.

18. Yoshikawa, M.; Kato, T.; KTakenishi, T. Tetrahedron Lett. 1967, 5065.

19. Chaput, J. C.; Yu, H.; Zhang, S. Chem. Biol. 2012, 19, 1360. 


\section{Graphical Abstract}

To create your abstract, type over the instructions in the template box below.

Fonts or abstract dimensions should not be changed or altered.

A One-Pot Synthesis of $\alpha$-L-threofuranosyl
nucleoside triphosphates (tNTPs)
Sujay $\mathrm{P}$. Sau and John C. Chaput

